\title{
Effects of Operational Frequency Scaling in Multi-Degree of Freedom MEMS Gyroscopes
}

\author{
Adam R. Schofield, Student Member, IEEE, Alexander A. Trusov, Student Member, IEEE, and
} Andrei M. Shkel, Member, IEEE

\begin{abstract}
This paper analyzes the design tradeoffs associated with increasing the operational frequency of single-axis microelectromechanical systems (MEMS) gyroscopes with multi-degree of freedom (DOF) sense modes. Previously, a $z$-axis multi-DOF gyroscope (1-DOF drive, 2-DOF sense) was shown to be robust to thermal variations using a prototype with a subkilohertz operational frequency; automotive applications, however, require higher frequencies of operation to suppress the effect of ambient vibrations. To study scaling effects on the multi-DOF concept, design equations were obtained in terms of operational frequency. These revealed a constraint on system parameters that introduces two scaling methods that dictate a tradeoff between gain, die size, and sense capacitance. Second generation multi-DOF gyroscopes were designed and fabricated resulting in 0.7-, 3.1-, and 5.1-kHz devices with smaller sense mode resonant frequency spacings than previously achievable. Experimental rate characterization resulted in scale factors of $14.2,5.08$, and $2.34 \mu \mathrm{V} / \% / \mathrm{s}$, respectively, confirming the predicted scaling effects while also demonstrating the feasibility of increased frequency multi-DOF gyroscopes.
\end{abstract}

\section{INTRODUCTION}

V IBRATORY micromachined gyroscopes operate based on the Coriolis effect, where a rotation-induced energy transfer occurs between two vibrational modes; conventionally, these modes, commonly referred to as drive and sense, are realized as single degree of freedom (DOF) dynamic systems using either linear or angular motion [1]. When the drive and sense resonant frequencies are equal, or mode-matched, the sensor output can be increased proportionally to the quality factor resulting in larger gain at the cost of reduced mechanical bandwidth [2]. Maintaining this matching condition, however, is difficult, especially in the presence of environmental variations, so typically, the frequencies are designed with some prescribed mismatch [3].

Structural designs that provide inherent robustness to environmental variations are an option to consider for automotive grade devices, where reducing the sensitivity of the device to changes in temperature is critical [4], [5]. Several concepts

Manuscript received March 17, 2008; revised May 23, 2008; accepted May 24,2008 . This work was supported by the Custom Sensors and Technologies (formerly BEI Technologies) Systron Donner Automotive Division under Contract BEI-36974 and by the University of California Discovery program ELE0410202. The associate editor coordinating the review of this manuscript and approving it for publication was Dr. Subhas Mukhopadhyay.

The authors are with the Department of Mechanical and Aerospace Engineering, University of California, Irvine, Irvine, CA 92697 USA (e-mails: adam. schofield@uci.edu; atrusov@uci.edu; ashkel@uci.edu).

Color versions of one or more of the figures in this paper are available online at http://ieeexplore.ieee.org.

Digital Object Identifier 10.1109/JSEN.2008.2004299 were proposed to improve robustness by increasing the number of mechanical degrees of freedom in the drive mode [6], [7]. The increased robustness of these devices, however, is achieved using nonresonant actuation that will ultimately lead to smaller drive mode amplitudes or larger driving voltages. An alternative multi-DOF design with a conventional 1-DOF drive mode and a 2-DOF sense mode was introduced in [8]. This approach is more suitable for high-amplitude, closed-loop drive operation while still taking advantage of the robustness improvements of the multi-DOF sense system.

The initial demonstration of the multi-DOF gyroscope concept reported angular rate sensors robust to fabrication imperfections and changes in temperature and pressure using a prototype with a subkilohertz operational frequency [8]. Typical operating conditions for automotive applications, however, require much higher operational frequencies (generally greater than 10 $\mathrm{kHz}$ ) to suppress the effect of environmental vibrational noise [3], [4]. For conventional gyroscope designs with 1-DOF drive and sense modes, increasing the resonant frequencies of the modes to meet the specification is straightforward; increasing the operational frequency of the multi-DOF gyroscope, however, is more complex due to the increased number of coupled parameters defining the overall dynamics.

This paper analyzes the tradeoffs associated with increasing the frequency of multi-DOF gyroscopes. Because the drive mode is a conventional 1-DOF dynamic system, the major focus of this work is on the scaling effects on the 2-DOF sense mode. The multi-DOF concept is introduced in Section II and sense mode design equations as well as expressions for both gain and bandwidth in terms of structural parameters are presented in Section III. These are used in Section IV to show the effects of operational frequency scaling on the multi-DOF design leading to the redesign of prototypes discussed in Section V. Section VI presents the fabrication and characterization of redesigned multi-DOF prototypes, and finally, Section VII contains concluding remarks.

\section{Multi-DOF CONCEPT}

The multi-DOF gyroscope concept addresses the issue of robustness in conventional gyroscopes by increasing the number of sense mode DOF [8]. The mechanical structure of the gyroscope consists of a single DOF drive mode and a 2-DOF sense mode as shown schematically in Fig. 1. The design employs mechanical mode decoupling where the two sense masses are suspended inside of a drive frame in order to minimize quadrature. The decoupling frame is constrained so that it only oscillates in the drive direction together with the sense masses, while the 


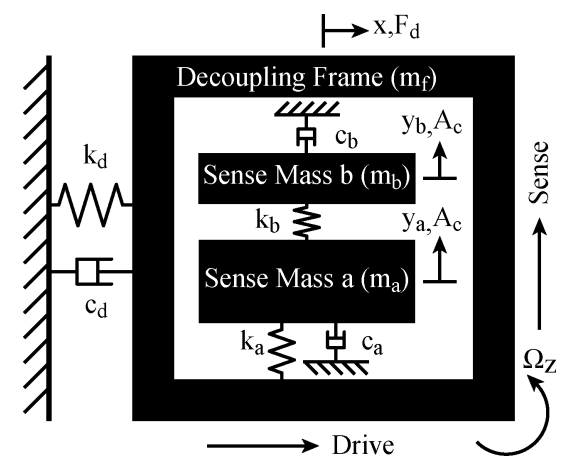

Fig. 1. Lumped structural model of the multi-DOF gyroscope (1-DOF drive, 2-DOF sense).

sense masses are constrained to vibrate only in the sense direction relative to the frame. Thus, the ideal system can be represented by the following simplified equations

$$
\begin{aligned}
\ddot{x}+\frac{c_{d}}{M_{d}} \dot{x}+\frac{k_{d}}{M_{d}} x & =\frac{F_{d}}{M_{d}} \\
\ddot{y}_{a}+\frac{c_{a}}{m_{a}} \dot{y}_{a}+\left(\frac{k_{a}+k_{b}}{m_{a}}\right) y_{a}-\frac{k_{b}}{m_{a}} y_{b} & =-2 \dot{x} \Omega_{z} \\
\ddot{y}_{b}+\frac{c_{b}}{m_{b}} \dot{y}_{b}+\frac{k_{b}}{m_{b}} y_{b}-\frac{k_{b}}{m_{b}} y_{a} & =-2 \dot{x} \Omega_{z}
\end{aligned}
$$

where the drive direction is along the $x$-axis, the sense direction is along the $y$-axis, and the input angular rate is along the $z$-axis [9]. The Coriolis term in (1) was neglected because feedback is typically employed to keep the drive amplitude constant. The angular acceleration and centrifugal terms in (2) and (3) were neglected because the input rates $(<5 \mathrm{~Hz})$ and frequency $(<$ $50 \mathrm{~Hz}$ ) of the rotational inputs are much smaller than typical resonant frequencies $(>1 \mathrm{kHz})$.

The drive mode of the multi-DOF gyroscope is described by (1), which consists of the decoupling frame and both sense masses moving in unison along the drive direction due to a harmonic forcing input $F_{d}$ applied to the frame. The drive mode is a single DOF system where the natural frequency is a function of the total drive mode mass $M_{d}=m_{f}+m_{a}+m_{b}$ and the total drive mode stiffness $k_{d}$. The drive mode damping coefficient $c_{d}$ consists of the total energy loss due to the drive mode motion of all the moving masses.

The sense mode is described by (2) and (3) in terms of the sense direction displacements of both sense masses $y_{a}$ and $y_{b}$ that are forced into vibration in the presence of an angular rate $\Omega_{z}$. The 2-DOF system has two resonant frequencies that are functions of the sense masses $m_{a}$ and $m_{b}$ as well as the sense mode stiffnesses $k_{a}$ and $k_{b}$. The sense mode damping coefficients $c_{a}$ and $c_{b}$ arise due to the motion of the sense masses in the sense direction relative to the fixed substrate; damping between the masses has been neglected due to the much smaller substrate spacing $(\approx 5 \mu \mathrm{m})$ compared to the sense mass separation $(\approx 30 \mu \mathrm{m})$.

A simulated frequency response of the multi-DOF gyroscope with superimposed drive and sense modes is shown in Fig. 2. The drive mode resonant frequency is designed to be situated within the wide area of relatively constant gain between the two

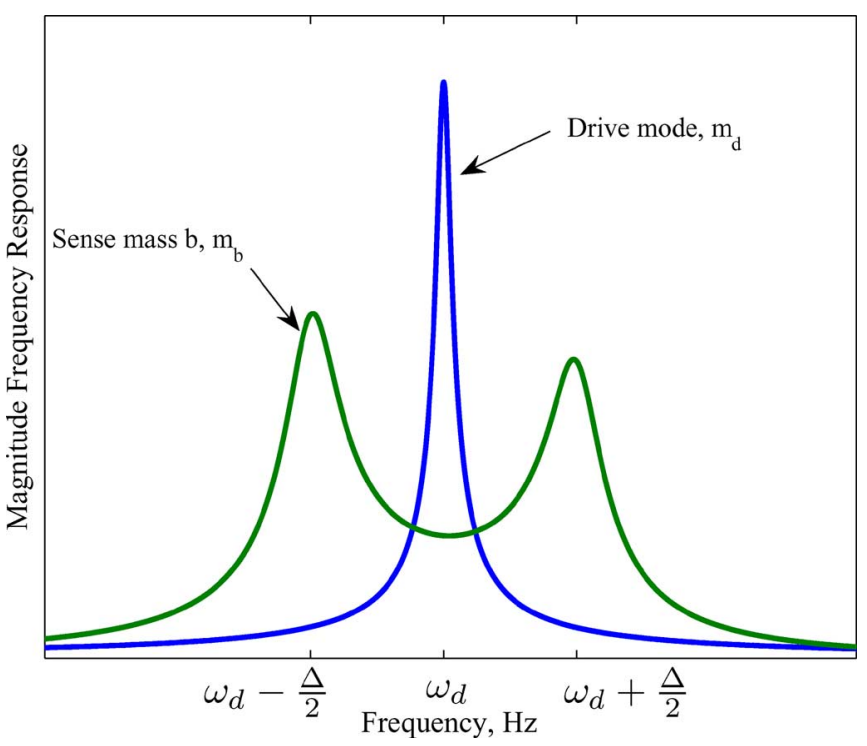

Fig. 2. Simulated drive and sense mode frequency response of the multi-DOF gyroscope with 2-DOF sense mode.

sense mode resonances. It is this flat region in the sense mode frequency response that provides the multi-DOF gyroscope with improved robustness and bandwidth by mechanical design. By operating the device in this region, one takes advantage of the nonresonant sense response, which is stable in amplitude over a wide frequency range.

\section{Multi-DOF Design AND ANALYsis}

For conventional gyroscopes, the main mechanical design parameters are the desired 1-DOF drive and sense resonant frequencies because the relative location of these frequencies ultimately determines the mechanical gain and bandwidth of the sensor [3]. On the other hand, multi-DOF gyroscopes have an expanded design space due to the presence of an additional mass in the 2-DOF sense mode. Therefore, the multi-DOF concept is characterized by three frequencies: the drive mode resonance $\omega_{d}$ and the lower and higher sense mode resonances $\omega_{1}$ and $\omega_{2}$, respectively. The mechanical design required to obtain these frequencies as well as their relationship to the mechanical gain and bandwidth of the multi-DOF device is discussed in the following sections.

\section{A. Design Equations}

As mentioned above, the drive mode of the multi-DOF gyroscope is a 1-DOF dynamic system where the operational frequency $\omega_{d}$ can be achieved independent of the sense mode by adjusting the drive mode stiffness $k_{d}$ or the decoupling frame mass $m_{f}$ (see Fig. 1). Therefore, the major focus of multi-DOF design is on the 2-DOF sense mode, specifically on identifying the structural parameters $k_{a}, k_{b}, m_{a}$, and $m_{b}$ needed to realize the desired resonant frequencies $\omega_{1}$ and $\omega_{2}$.

The locations of these frequencies, however, are not independent because the drive mode is designed to be in the region of constant amplitude in the sense mode frequency response as shown in Fig. 2. If the drive mode resonance is assumed to be 
equally spaced from the sense mode peaks, the desired sense mode resonant frequencies can be expressed as

$$
\omega_{1,2}=\omega_{d} \mp \frac{\Delta}{2}
$$

where $\omega_{d}$ is the operational frequency and $\Delta$ is the sense mode peak spacing, defined as $\Delta=\omega_{2}-\omega_{1}$. Thus, the three characteristic frequencies of the multi-DOF concept can be determined solely using two quantities: operational frequency $\omega_{d}$ and sense mode peak spacing $\Delta$. The goal is now to obtain sense mode equations for the structural parameters required to obtain resonant frequencies given by (4) for specified design parameters.

From (2) and (3), the following structural frequencies can be defined:

$$
\omega_{a}^{2}=\frac{k_{a}+k_{b}}{m_{a}} \quad \omega_{b}^{2}=\frac{k_{b}}{m_{b}} \quad \omega_{c}^{2}=\frac{k_{b}}{\sqrt{m_{a} m_{b}}}
$$

where $\omega_{a}$ and $\omega_{b}$ are the frequencies of the system if the larger mass $m_{a}$ and the smaller mass $m_{b}$ (shown in Fig. 1) are held clamped, respectively, while $\omega_{c}$ is a term that expresses the strength of the coupling between the two masses [10]. Using the quantities in (5), the eigenvalue equation for the 2-DOF sense mode becomes

$$
\omega^{4}-\omega^{2}\left(\omega_{a}^{2}+\omega_{b}^{2}\right)+\left(\omega_{a}^{2} \omega_{b}^{2}-\omega_{c}^{4}\right)=0
$$

which has the following solutions:

$$
\omega_{1,2}^{2}=\frac{1}{2}\left(\omega_{a}^{2}+\omega_{b}^{2} \mp \sqrt{\omega_{a}^{4}+\omega_{b}^{4}-2 \omega_{a}^{2} \omega_{b}^{2}+4 \omega_{c}^{4}}\right) .
$$

The solution of the 2-DOF eigenvalue equation (6) defines the sense mode resonant frequencies $\omega_{1}$ and $\omega_{2}$ in terms of the structural frequencies $\omega_{a}, \omega_{b}$, and $\omega_{c}$. Substituting (4) into (6) and solving simultaneously for $\omega_{a}$ and $\omega_{b}$ gives

$$
\begin{aligned}
& \omega_{a}^{2}=\omega_{d}^{2}+\left(\frac{\Delta}{2}\right)^{2} \pm \sqrt{\omega_{d}^{2} \Delta^{2}-\omega_{c}^{4}} \\
& \omega_{b}^{2}=\omega_{d}^{2}+\left(\frac{\Delta}{2}\right)^{2} \mp \sqrt{\omega_{d}^{2} \Delta^{2}-\omega_{c}^{4}}
\end{aligned}
$$

which are sense mode design equations for the structural frequencies $\omega_{a}$ and $\omega_{b}$ in terms of the operational frequency $\omega_{d}$, sense mode peak spacing $\Delta$, and the coupling parameter $\omega_{c}$.

The dependence of (7) and (8) on the coupling parameter $\omega_{c}$ can be eliminated by examining (5) and noting the following relationship:

$$
\mu \omega_{b}^{2}=\omega_{c}^{2}
$$

which defines the direct dependence of $\omega_{c}$ on $\omega_{b}$ by the proportionality constant $\mu$. This factor is an important coupled system parameter known as the mass ratio and is defined as

$$
\mu^{2}=\frac{m_{b}}{m_{a}}
$$

where $m_{b}$ is the smaller sense mass and $m_{a}$ is the larger sense mass. Substituting (9) into (7) and (8) gives

$$
\begin{aligned}
& \omega_{a}^{2}= \frac{\omega_{d}^{2}+\left(\frac{\Delta}{2}\right)^{2}+2 \mu^{2}\left(\omega_{d}^{2}+\left(\frac{\Delta}{2}\right)^{2}\right)}{1+\mu^{2}} \\
& \mp \frac{\sqrt{\omega_{d}^{2} \Delta^{2}-\mu^{2}\left(\omega_{d}+\frac{\Delta}{2}\right)^{2}\left(\omega_{d}-\frac{\Delta}{2}\right)^{2}}}{1+\mu^{2}} \\
& \omega_{b}^{2}=\frac{\omega_{d}^{2}+\left(\frac{\Delta}{2}\right)^{2} \pm \sqrt{\omega_{d}^{2} \Delta^{2}-\mu^{2}\left(\omega_{d}+\frac{\Delta}{2}\right)^{2}\left(\omega_{d}-\frac{\Delta}{2}\right)^{2}}}{1+\mu^{2}}
\end{aligned}
$$

which are sense mode design equations for the structural frequencies $\omega_{a}$ and $\omega_{b}$ in terms of the drive frequency $\omega_{d}$, peak spacing $\Delta$, and mass ratio $\mu^{2}$.

Examination of (11) and (12), however, reveals the existence of a constraint for real systems

$$
\omega_{d}^{2} \Delta^{2}-\mu^{2}\left(\omega_{d}+\frac{\Delta}{2}\right)^{2}\left(\omega_{d}-\frac{\Delta}{2}\right)^{2}>0
$$

which is dependent on operational frequency, peak spacing, and mass ratio. Therefore, these parameters cannot be specified arbitrarily, but rather must be chosen to ensure the constraint is met. From a design perspective, this means that combinations of mass ratios, peak spacings, and operational frequencies must be checked using (13) to determine if they are valid. If verified, the suspensions required to achieve the desired sense mode resonant frequencies can be found using (11) and (12) assuming the value of the sense masses are known.

\section{B. Sense Mode Gain and Bandwidth}

As mentioned above, the multi-DOF gyroscope takes advantage of the nearly constant amplitude between the resonances in the sense mode frequency response, as shown above in Fig. 2. While this nonresonant region provides the device with increased robustness and bandwidth at the cost of reduced gain, it also makes it more difficult to determine how the operational frequency $\omega_{d}$, peak spacing $\Delta$, and damping affect the mechanical characteristics of the sensor.

Simulations were used to investigate the response of the 2-DOF sense mode to changes in damping conditions. Using the dynamic equations (2) and (3), frequency responses were obtained and presented in Fig. 3 for the amplitude of the smaller mass $m_{b}$ at various mass normalized damping coefficients. As the damping is decreased, the amplitude in the operational region approaches the zero-damping case while the bandwidth, calculated as a 3-dB increase from the minimum gain between the resonant peaks, slightly decreases as indicated by the markers. Thus, much like conventional devices, a tradeoff exists between bandwidth and gain depending on the pressure at which the device is operating.

Fig. 3 also reveals that limits of maximum gain and minimum bandwidth in the operational region can be determined by examining the 2-DOF system at zero damping. Using these asymptotic values, the effect of design parameters on the mechanical characteristics can be determined using the sense mode transfer 


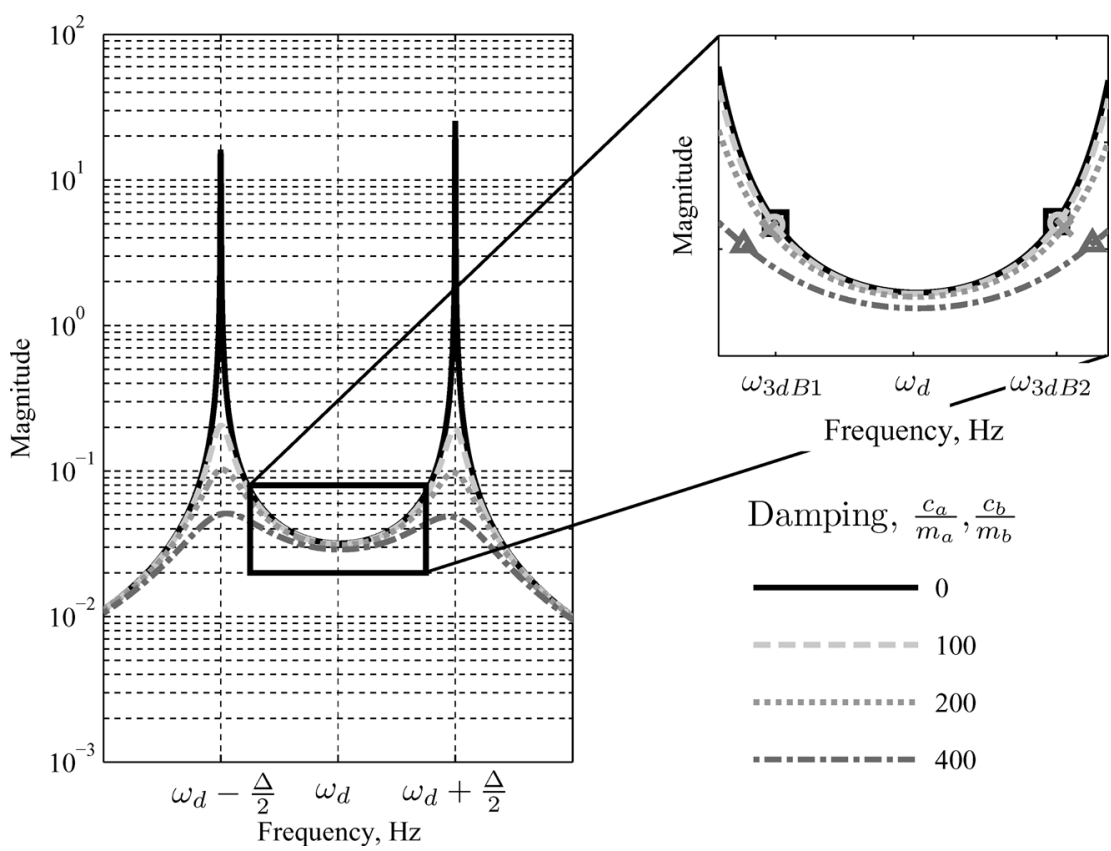

Fig. 3. Simulated frequency responses for various damping coefficients with markers indicating the 3-dB bandwidth points.

functions. Starting from (2) and (3), the following transfer functions can be found:

$$
\begin{aligned}
& H_{a}(s)=\frac{s^{2}+\left(1+\mu^{2}\right) \omega_{b}^{2}}{s^{4}+\left(\omega_{b}^{2}+\omega_{a}^{2}\right) s^{2}-\mu^{2} \omega_{b}^{4}+\omega_{a}^{2} \omega_{b}^{2}} \\
& H_{b}(s)=\frac{s^{2}+\omega_{a}^{2}+\omega_{b}^{2}}{s^{4}+\left(\omega_{b}^{2}+\omega_{a}^{2}\right) s^{2}-\mu^{2} \omega_{b}^{4}+\omega_{a}^{2} \omega_{b}^{2}}
\end{aligned}
$$

where the input is Coriolis acceleration applied to both masses and the output is the displacement of sense mass $m_{a}$ [see (14)] and sense mass $m_{b}$ [see (15)], assuming zero damping.

Despite having two transfer functions, one for each sense mass, the gain and bandwidth of a multi-DOF device are dependent on only the magnitude of the smaller mass $m_{b}$, because it is used to detect the Coriolis signal. The reason for using the smaller mass versus the larger mass for sensing is due to the location of the antiresonance $\omega_{a}$ in the frequency response of mass $m_{a}$, which is defined as the frequency at which the numerator of (14) is equal to zero. It can be shown that $\omega_{1}^{2}<\omega_{a}^{2}<\omega_{2}^{2}$, which means the antiresonant frequency of the larger mass $m_{a}$ is always between the sense mode resonant frequencies. Therefore, the amplitude of the smaller mass $m_{b}$ is maximized relative to the larger mass in the operational region of the device making it the optimum mass for sensing motion induced by angular rotations. Substituting (11), (12), and $s=j \omega$ (where $j$ is the imaginary number) into (15) and taking the absolute value gives

$$
G_{b}(\omega)=\frac{\omega^{2}-2 \omega_{d}^{2}-\frac{\Delta^{2}}{2}}{\left(\omega^{2}-\left(\omega_{d}-\frac{\Delta}{2}\right)^{2}\right)^{2}\left(\omega^{2}-\left(\omega_{d}+\frac{\Delta}{2}\right)^{2}\right)^{2}}
$$

which is the transfer function magnitude of sense mass $m_{b}$ for input frequencies $\omega$ assuming $\omega_{1}<\omega<\omega_{2}$.

Because (16) gives the mechanical gain for any frequency between the resonances, it can be used to determine the sense mode gain of a multi-DOF device. The sense mode gain, defined as the amplitude of $m_{b}$ at the operational frequency $G_{b}\left(\omega_{d}\right)$, is of particular interest because this will ultimately determine the scale factor of the device. Substituting $\omega=\omega_{d}$ into (16) gives

$$
G_{b}\left(\omega_{d}\right)=\frac{\omega_{d}^{2}+\frac{\Delta^{2}}{4}}{\left(\omega_{d}^{2}-\frac{\Delta^{2}}{4}\right) \Delta^{2}}
$$

which is the sense mode gain in terms of both the peak spacing and the desired operational frequency. If the peak spacing is assumed to be much smaller than the operational frequency $\omega_{d} \gg$ $\Delta$, (17) reduces to

$$
G_{b}\left(\omega_{d}\right) \approx \frac{1}{\Delta^{2}}
$$

which is typical for most 2-DOF sense systems. Therefore, it can be concluded from (18) that the major parameter affecting the sense mode gain of multi-DOF devices is the peak spacing, where smaller spacings result in larger gain values.

The sense mode bandwidth of a multi-DOF device is defined by the frequencies at which the amplitude increases by $3 \mathrm{~dB}$ versus the minimum value between the resonances. The frequency at which this minimum gain occurs can be found by differentiating (16), setting it equal to zero, and solving for frequency which gives

$$
\omega_{\min }=\sqrt{\omega_{d}^{2}+\frac{3 \Delta^{2}}{4}}
$$

where $\omega_{\min }$ is the minimum gain frequency. Substituting (19) into (16) gives

$$
G_{\omega_{\min }}=\frac{1}{\Delta^{2}}
$$

which is the minimum gain value in the sense mode operational region. 
It should be noted that the sense mode gain (17) and the minimum gain (20) are different. This is because the minimum gain frequency (19) is not equal to the operational frequency, but rather dependent on both operational frequency and peak spacing. This means that the minimum gain point in the operational region is not equally spaced from the resonances as assumed for the drive frequency; instead, it is shifted to slightly higher frequencies as the peak spacing is increased. However, for typical 2-DOF systems where the peak spacing is much smaller than the operational frequency, the sense mode gain (18) and the minimum gain (20) can be assumed equal.

The bandwidth can be determined using (20) by solving for the frequencies at which the amplitude increases by $\sqrt{2}$ according to

$$
G_{b}\left(\omega_{3 \mathrm{~dB}}\right)=\frac{\sqrt{2}}{\Delta^{2}}
$$

where $\omega_{3} \mathrm{~dB}$ are the two $3-\mathrm{dB}$ frequency points. Solving (21) gives

$$
\begin{aligned}
& \omega_{3 \mathrm{~dB} 1,2}^{2}= \omega_{d}^{2}+(1+\sqrt{2})\left(\frac{\Delta}{2}\right)^{2} \\
& \mp \sqrt{\left(1-\frac{\sqrt{2}}{2}\right) \omega_{d}^{2}+2(1-\sqrt{2})\left(\frac{\Delta}{2}\right)^{4}}
\end{aligned}
$$

where $\omega_{3} \mathrm{~dB} 1$ and $\omega_{3} \mathrm{~dB} 2$ are the lower and higher 3-dB frequencies, respectively. Together, the 3-dB points from (22) define the bandwidth of the 2-DOF sense system according to $B W_{3 \mathrm{~dB}}=$ $\omega_{3 \mathrm{~dB} 2}-\omega_{3 \mathrm{~dB} 1}$. Again, if the peak spacing is assumed to be much smaller than the operational frequency $\omega_{d} \gg \Delta$, the bandwidth simplifies to

$$
B W_{3 \mathrm{~dB}}=\left(\sqrt{1-\frac{\sqrt{2}}{2}}\right) \Delta \approx 0.5412 \Delta
$$

which corresponds to a 3-dB bandwidth of roughly half of the peak spacing independent of operational frequency.

\section{FREQUENCY SCALING}

As mentioned above, it is desirable to have increased operational frequencies in order to suppress the effect of ambient vibrations on the gyroscope [3], [4]. For conventional devices, increasing the operational frequency of the device is straightforward because both the drive and sense modes are 1-DOF systems; increasing the frequency of multi-DOF gyroscopes, however, is not trivial due to the increased design space of the sense mode.

To address multi-DOF design complexity, equations were derived in the previous section for the stiffnesses required to achieve the specified sense mode resonant frequencies. These equations, however, revealed the existence of a constraint (13) meaning that the desired structural parameters have to be verified to ensure they result in a physically realizable system. When viewed from a scaling perspective, this implies that the peak spacing and/or mass ratio may have to be adjusted as the operational frequency of the device is increased in order to meet the constraint. This gives rise to the two methods of scaling discussed below.

\section{A. Constant Mass Ratio}

An intuitive method for increasing the operational frequency of multi-DOF gyroscopes is to adjust the stiffnesses while leaving the masses unchanged so that the mass ratio remains constant. The effect of constant mass ratio frequency scaling on the 2-DOF system parameters can be seen by solving (13) for the peak spacing

$$
\Delta>\frac{2}{\mu}\left(\sqrt{1+\mu^{2}}-1\right) \omega_{d}=\Delta_{\min }
$$

where $\Delta_{\min }$ is defined as the minimum achievable peak spacing. For small mass ratios $\mu^{2} \ll 1$, (23) can be approximated by

$$
\Delta>\Delta_{\min } \approx \mu \omega_{d}
$$

\section{B. Constant Peak Spacing}

The alternative method of increasing the operational frequency of a multi-DOF gyroscope is to maintain a constant peak spacing so that the sense mode gain of the system remains unchanged. The effect on the 2-DOF sense mode parameters can be seen by solving (13) for the mass ratio

$$
\mu^{2}<\frac{\omega_{d}^{2} \Delta^{2}}{\left(\omega_{d}+\frac{\Delta}{2}\right)^{2}\left(\omega_{d}-\frac{\Delta}{2}\right)^{2}}=\mu_{c}^{2}
$$

where $\mu_{c}^{2}$ is defined as the critical mass ratio. Assuming $\omega_{d} \gg$ $\Delta$, (24) simplifies to

$$
\mu^{2}<\mu_{c}^{2} \approx \frac{\Delta^{2}}{\omega_{d}^{2}}
$$

\section{Effect of Frequency Scaling}

As shown above, both constant mass ratio and constant peak spacing scaling result in expressions that limit the range of available design parameters. When the mass ratio or peak spacing become small, these expressions converge to a single inequality

$$
\frac{\Delta}{\mu}>\omega_{d}
$$

which defines the frequency scaling relationship between the peak spacing and mass ratio.

Constant mass ratio scaling, while requiring little design modification, eventually necessitates an increase of peak spacing at higher frequencies according to (25). As the peak spacing becomes larger, the device will suffer a reduction of sense mode gain as shown by (18). The sense mode gain of the multi-DOF device can be preserved by maintaining a constant peak spacing while increasing the frequency; however, this results in a mandatory decrease of mass ratio. From (10), it can be seen that a reduction of mass ratio corresponds to a system where the larger mass $m_{a}$ gets larger, the smaller mass $m_{b}$ gets smaller, or both. Ultimately, this mass ratio change results in a device with a larger die size to accommodate the bigger mass 


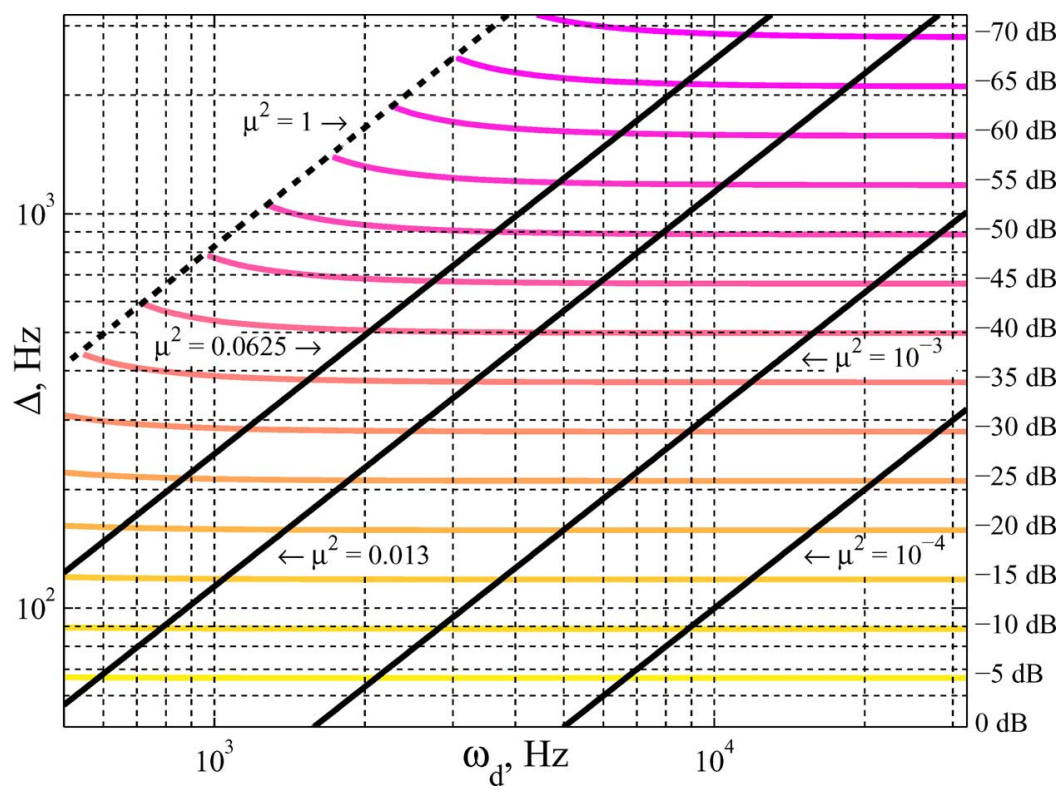

Fig. 4. Change in gain and mass ratio required for combinations of operational frequency and peak spacing.

and/or a decrease in sense capacitance to minimize the smaller mass.

Thus, the main effect of operational frequency scaling in multi-DOF structures represented by Fig. 1 is this tradeoff between the sense mode gain defined by the peak spacing and the die size/sense capacitance determined by the mass ratio. This relationship is presented graphically in Fig. 4, which was generated using combinations of operational frequency ( $x$-axis) and peak spacing ( $y$-axis) to calculate the sense mode gain of the system (17) as well as the critical mass ratio required to achieve the design (24). The results are plotted as contour lines of constant mass ratios for various indicated values and constant sense mode gains with corresponding numbers indicating the decibel reduction normalized to the largest simulated value.

Constant mass ratio frequency scaling is represented in Fig. 4 by following the lines of constant mass ratio. As the operational frequency is increased, the corresponding minimum peak spacing becomes larger resulting in a reduction of sense mode gain. Increasing operational frequency while maintaining a constant peak spacing corresponds to horizontal lines in Fig. 4. While there is little change in the sense mode gain (especially when $\omega_{d} \gg \Delta$ ), the major drawback is that the mass ratio required to achieve this design decreases ultimately resulting in die size adjustments.

\section{SECOND GENERATION REDESIGN}

The initial demonstration of the multi-DOF concept was performed on a prototype with a mass ratio of $\mu^{2}=0.0625$, an operational frequency of $750 \mathrm{~Hz}$, and a peak spacing of $250 \mathrm{~Hz}$ [8]. From Fig. 4, it can be seen that increasing the frequency of the original design without altering its mass ratio would result in peak spacings of more than $1200 \mathrm{~Hz}$ at an operational frequency of $5 \mathrm{kHz}$ and $2500 \mathrm{~Hz}$ at $10 \mathrm{kHz}$.

A second generation of multi-DOF devices was designed to improve the frequency scaling characteristics of the sensors. The redesigned prototypes were realized with a mass ratio of
TABLE I

Parameters of the SECOND Generation Multi-DOF DEVICES AND PREVIOUS PROTOTYPE

\begin{tabular}{lcccc}
\hline & $\begin{array}{c}\text { Previous } \\
{[8]}\end{array}$ & $\begin{array}{c}\text { Device } \\
\# 1\end{array}$ & $\begin{array}{c}\text { Device } \\
\# 2\end{array}$ & $\begin{array}{c}\text { Device } \\
\# 3\end{array}$ \\
Drive mode & & & & \\
\hline Frequency, $\mathrm{kHz}$ & 0.75 & 0.7 & 3.1 & 5.1 \\
Capacitance, $\mathrm{pF}$ & 0.75 & 0.59 & 0.59 & 0.59 \\
\hline Sense mode & & & & \\
\hline Peak spacing, $\mathrm{Hz}$ & 250 & 150 & 400 & 600 \\
Mass ratio $\mu^{2}$ & 0.0625 & 0.013 & 0.013 & 0.013 \\
Capacitance, $\mathrm{pF}$ & 1.16 & 0.51 & 0.51 & 0.51 \\
\hline
\end{tabular}

$\mu^{2}=0.013$ by reducing mass $m_{b}$ as well as increasing the size of mass $m_{a}$; this resulted in a new device that had an overall larger die size and reduced sense capacitance as compared to the previous implementation. The reduction in mass ratio, however, allows for smaller peak spacings at higher operational frequencies as shown in Fig. 4, which leads to gain improvements of over $10 \mathrm{~dB}$ versus the previous design.

The second generation devices were designed with iterated frequencies while keeping the mass ratio constant in order to experimentally characterize the scaling effects presented above. Three prototypes with operational frequencies of $0.7,3.1$, and $5.1 \mathrm{kHz}$ and peak spacings of 150,400 , and $600 \mathrm{~Hz}$, respectively, were chosen for the analysis. A summary of device parameters for both the original and second generation designs used in this work are presented in Table I.

\section{EXPERIMENTAL CHARACTERIZATION}

\section{A. Fabrication}

The fabrication of the redesigned multi-DOF devices was done using an inhouse wafer scale silicon-on-insulator (SOI) process. SOI wafers with a conductive $75-\mu \mathrm{m}$-thick device layer and 5- $\mu \mathrm{m}$ buried oxide were used. Shipley 1827 photoresist was patterned using a chrome-on-glass photomask and a Karl 


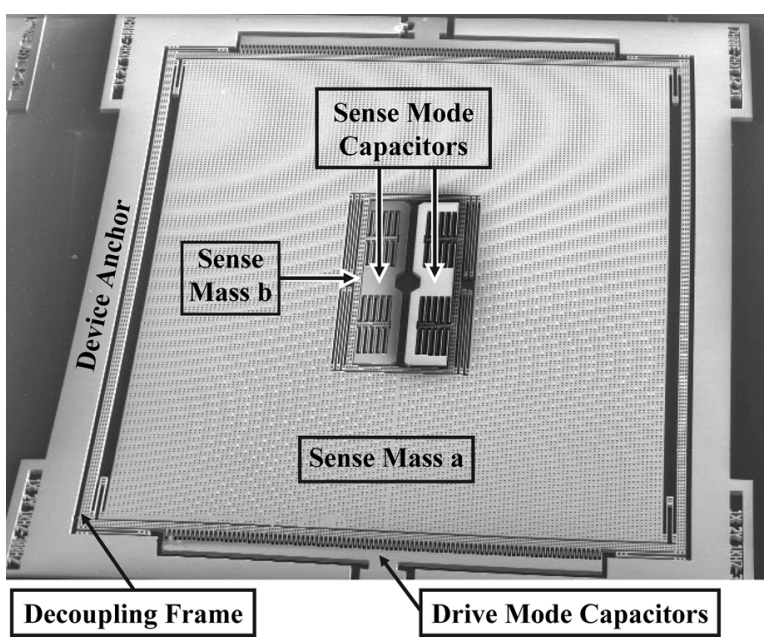

Fig. 5. SEM image of a fabricated multi-DOF gyroscope with 2-DOF sense mode.

Suss MA6 exposure system. After photoresist development, the wafers were subjected to a deep reactive ion etching (DRIE) using a surface technology systems (STS) advanced silicon etching (ASE) tool. The fabricated SOI wafers were diced and individual devices were packaged and wirebonded.

Fig. 5 shows a scanning electron microscope (SEM) image of a fabricated device. The devices have lateral comb electrodes in the drive mode for both actuation and sensing. The motion of the smaller sense mass is detected using parallel plate capacitors with capacitive gaps of $10 \mu \mathrm{m}$.

\section{B. Experimental Setup}

The actuation and detection scheme used to characterize the performance of the fabricated gyroscopes is shown in Fig. 6. The combination of a direct current (dc) bias with an alternating current (ac) driving voltage at the drive mode resonant frequency was applied to the fixed driving electrodes. Capacitive coupling from drive to sense results in a parasitic current corrupting the motional signal; separation of the useful signal from the feed-through is accomplished using electromechanical amplitude modulation (EAM), where a carrier ac voltage is applied to the movable mass resulting in the amplitude modulation (AM) of the motional signal. Two demodulations, first at the carrier frequency and then at the drive frequency, are used to extract the motional signal from the total EAM pickup signal. A detailed description of the signal processing can be found, for example, in [11].

In this paper, two AMETEK Advanced Measurement Technology Signal Recovery (Oak Ridge, TN) Model 7265 digital lock-in amplifiers were used to generate the drive and the carrier ac voltages and perform the two stages of demodulation. The devices were characterized at constant angular rates using an Ideal Aerosmith (East Grand Forks, MN) 1291BR rate table. All of the experiments were performed at atmospheric pressure and room temperature.

\section{Angular Rate Characterization}

The redesigned multi-DOF gyroscopes were characterized using constant angular rotations in the range of $\pm 1000 \%$ s; the

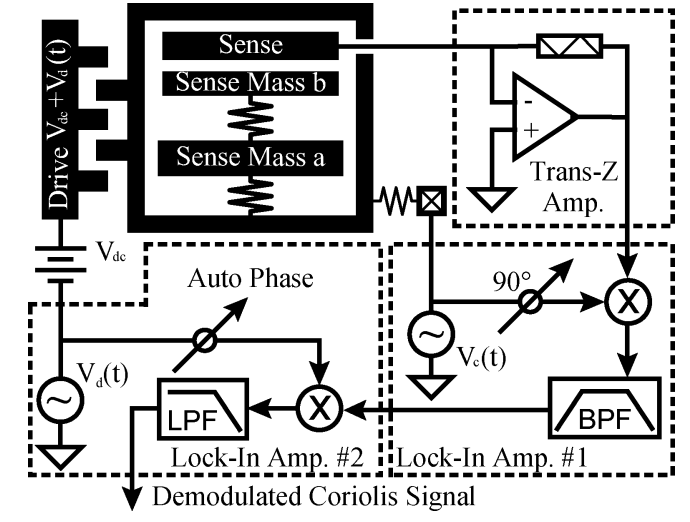

Fig. 6. Schematic of the open-loop actuation and EAM-based detection for gyroscope characterization.

experimental data for all three devices is plotted in Fig. 7. The scale factor of each device, noted in the legend, was determined using a least squares fit of the data and was used to generate the plotted trendlines. The linearity of the lowest frequency device was $0.5 \%$ full scale output (FSO), while the linearity of the two higher frequency devices was $1.5 \%$ FSO. The degradation was due to the significant driving voltages required for the higher frequency devices, leading to increased electrical noise levels at the sense mode pickup.

The lowest frequency device, $0.7-\mathrm{kHz}$ drive frequency and $150-\mathrm{Hz}$ sense spacing, closely matches the original multi-DOF prototype providing a comparison of the two designs. The scale factor of the low-frequency redesign was found to be $14.2 \mu \mathrm{V} /{ }^{\circ}$ $/ \mathrm{s}$, which is approximately half of the scale factor of $30 \mu \mathrm{V} / \% / \mathrm{s}$ reported in [8]. The scale factor reduction of the redesigned device can be attributed to slightly different driving amplitudes (5.8 versus $5 \mu \mathrm{m}$ ) as well as the $68 \%$ reduction of the sense mode capacitance used to reduce the mass ratio. The advantage of the redesign, however, is demonstrated by the experimentally obtained rate responses for the two higher frequency prototypes. The reduction of mass ratio ultimately allowed for smaller achievable peak spacings, limiting the reduction of sense mode gain at increased frequencies. Thus, a peak spacing of $600 \mathrm{~Hz}$ at $5.1 \mathrm{kHz}$ was realized leading to a device with a scale factor of $2.34 \mu \mathrm{V} / \%$ s.

Also, as the operational frequency is increased, a reduction of scale factor is apparent from Fig. 7; this is the expected result of the constant mass ratio scaling used to design the high-frequency prototypes. The scale factors of the three devices in decibels, normalized to the scale factor of the $0.7-\mathrm{kHz}$ prototype, are plotted in the inset of Fig. 7 to illustrate this effect. Increasing the frequency from 0.7 to $3.1 \mathrm{kHz}$ resulted in a scale factor reduction of $9 \mathrm{~dB}$ while an increase to $5.1 \mathrm{kHz}$ resulted in nearly a 16-dB drop. The 7-dB drop in scale factor from the $3.1-\mathrm{kHz}$ device with $400-\mathrm{Hz}$ spacing to the $5.1-\mathrm{kHz}$ device with $600-\mathrm{Hz}$ spacing agrees with the change in gain given by Fig. 4 while the change from the $0.7-\mathrm{kHz}$ device with $150-\mathrm{Hz}$ spacing to the $3.1-\mathrm{kHz}$ device is lower than predicted. The gain model, however, relies on a zero damping assumption leading to an overestimation of the actual scale factor. The discrepancy between the experimentally obtained scale factors in air presented in Fig. 7 


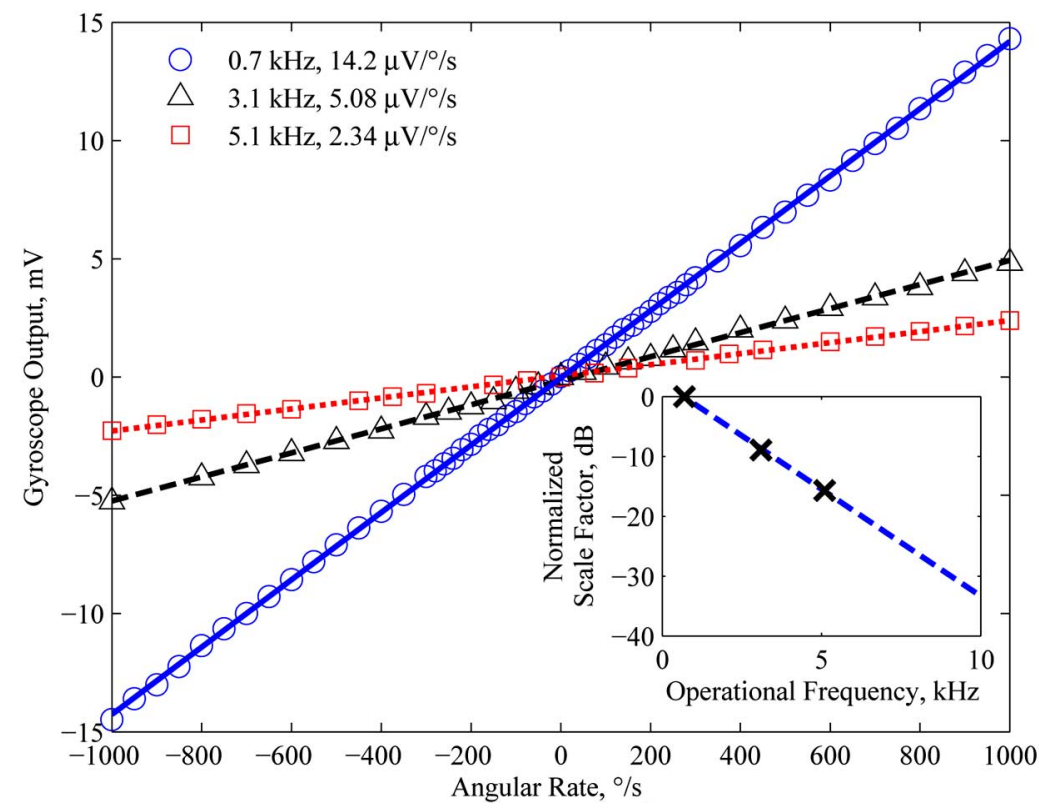

Fig. 7. Angular rate characterization of redesigned, second generation multi-DOF devices with measured scale factors. Inset: normalized scale factor of the three devices with fitted line to show the frequency scaling trend.

and the model becomes more pronounced at lower operational frequencies and smaller sense mode peak spacings.

\section{CONCLUSION}

Operational frequency scaling in multi-DOF gyroscopes was analyzed and experimentally characterized. Sense mode design equations were derived for the structural parameters required to realize the desired operational frequency and sense mode resonant peak spacing. A zero damping assumption was used to obtain expressions for the asymptotic maximum gain and minimum bandwidth of the sense mode. When the drive frequency is much higher than the separation of the sense mode resonances, both the gain and bandwidth are independent of the operational frequency.

The analysis revealed that the design parameters cannot be arbitrarily chosen, but rather must be verified to ensure they satisfy the derived constraint for physically realizable systems. This introduced two methods of multi-DOF frequency scaling, which ultimately limit the range of available parameters. Constant mass ratio scaling, while intuitive, results in the unavoidable increase of peak spacing, and correspondingly, a reduction in sense mode gain; constant peak spacing scaling, however, maintains the sense mode gain at the cost of a decrease in mass ratio. Thus, increasing the operational frequency of multi-DOF devices dictates a tradeoff between gain and die size/sense capacitance. Recently, an alternative design concept has been proposed by this group, which addresses the scaling issue and eliminates the tradeoff between gain and operational frequency [12].

Second generation multi-DOF prototypes with smaller mass ratios were designed to improve the frequency scaling of the device. Three devices with operational frequencies of $0.7,3.1$, and $5.1 \mathrm{kHz}$ were fabricated and characterized at constant angular rates. The measured scale factors $14.2,5.08$, and $2.34 \mu \mathrm{V} / \% / \mathrm{s}$ decreased for increasing frequency as expected by the constant mass ratio scaling analysis. The demonstration of the 3.1- and
5.1-kHz devices, however, are the highest frequency multi-DOF devices demonstrated, and despite the scaling drawbacks, highlight the feasibility of achieving increased frequency devices for automotive applications.

\section{ACKNOWLEDGMENT}

The authors would like to thank the UCI Integrated Nanosystems Research Facility for fabrication assistance and C. Acar and L. Costlow of Systron Donner Automotive (Concord, CA) for the useful discussions.

\section{REFERENCES}

[1] A. M. Shkel, "Type I and type II micromachined vibratory gyroscopes," in Proc. IEEE/ION Position Locat. Navigat. Symp., San Diego, CA, Apr. 24-27, 2006, pp. 586-593.

[2] N. Yazdi, F. Ayazi, and K. Najafi, "Micromachined inertial sensors," Proc. IEEE, vol. 86, no. 8, pp. 1640-1659, Aug. 1998.

[3] M. Weinberg and A. Kourepenis, "Error sources in in-plane silicon tuning-fork MEMS gyroscopes," J. Microelectromech. Syst., vol. 15, no. 3, pp. 479-491, Jun. 2006.

[4] R. Neul, U.-M. Gomez, K. Kehr, W. Bauer, J. Classen, C. Doring, E. Esch, S. Gotz, J. Hauer, B. Kuhlmann, C. Lang, M. Veith, and R. Willig," Micromachined angular rate sensors for automotive applications," IEEE Sensors J., vol. 7, no. 2, pp. 302-309, Feb. 2007.

[5] J. A. Geen, S. J. Sherman, J. F. Chang, and S. R. Lewis, "Single-chip surface micromachined integrated gyroscope with $50 \mathrm{deg} / \mathrm{h}$ Allan deviation," IEEE J. Solid-State Circuits, vol. 37, no. 12, pp. 1860-1866, Dec. 2002.

[6] C. Acar and A. Shkel, "Nonresonant micromachined gyroscopes with structural mode-decoupling," IEEE Sensors J., vol. 3, no. 4, pp. 497-506, Aug. 2003.

[7] S.-H. Jeon, J.-Y. Lee, H.-K. Jung, H.-K. Chang, and Y.-K. Kim, "Twomass system with wide bandwidth for SiOG (silicon on glass) vibratory gyroscopes," in Proc. Int. Conf. Solid State Sens. Actuators Microsyst., Seoul, Korea, Jun. 5-9, 2005, pp. 539-542.

[8] C. Acar and A. Shkel, "Inherently robust micromachined gyroscopes with 2-DOF sense-mode oscillator," J. Microelectromech. Syst., vol. 15, no. 2, pp. 380-387, Apr. 2006.

[9] A. R. Schofield, A. A. Trusov, and A. M. Shkel, "Structural design trade-offs for MEMS vibratory rate gyroscopes with 2-DOF sense modes," in Proc. ASME Int. Design Eng. Tech. Conf., Las Vegas, NV, Sep. 4-7, 2007.

[10] J. P. Den Hartog, Mechanical Vibrations. New York: Dover, 1985. 
[11] A. A. Trusov and A. M. Shkel, "Capacitive detection in resonant MEMS with arbitrary amplitude of motion," J. Micromech. Microeng., vol. 17, no. 8, pp. 1583-1592, Jul. 2007.

[12] A. A. Trusov, A. R. Schofield, and A. M. Shkel, "New architectural design of a temperature robust MEMS gyroscope with improved gain-bandwidth characteristics," in Proc. Hilton Head Workshop Solid State Sens. Actuators Microsyst., Hilton Head, SC, Jun. 1-5, 2008, pp. $14-17$.

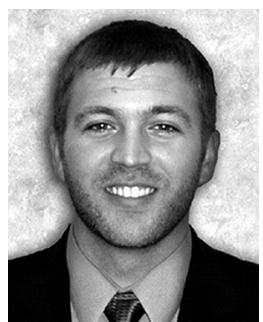

Adam R. Schofield (S'06) received the B.S. degree in mechanical engineering (summa cum laude) with honors and the M.S. degree in mechanical engineering (supported by an Ohio Space Grant Fellowship) from the University of Dayton, Dayton, $\mathrm{OH}$, in 2002 and 2005, respectively, Currently, he is working towards the Ph.D. degree in mechanical and aerospace engineering at the University of California, Irvine, where he focuses on the design, fabrication, and characterization of MEMS inertial sensors.

Mr. Schofield was awarded the Class of 2002 Award for Outstanding Mechanical Engineering Achievement by the University of Dayton. He is a member of Pi Tau Sigma, Tau Beta Pi, Golden Key Honor Society, and the American Society of Mechanical Engineers (ASME)

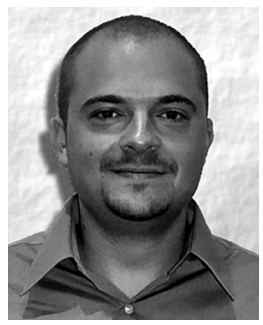

Alexander A. Trusov (S'06) received the diploma in applied mathematics and mechanics from Moscow State University, Moscow, Russia in 2004 and the M.S. degree in mechanical and aerospace engineering from University of California, Irvine, in 2006, where he is currently working towards the $\mathrm{Ph} . \mathrm{D}$. degree in mechanical and aerospace engineering.

His research interests include design, fabrication, and characterization of inertial MEMS and development of novel actuation, detection, signal processing, and control schemes for dynamic MEMS.

Mr. Trusov served as a reviewer for major journals in the fields of MEMS and sensors and is a member of the American Society of Mechanical Engineers (ASME).

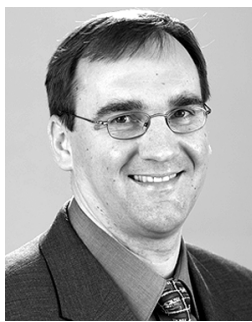

Andrei M. Shkel (S'95-M'98) received the diploma (with excellence) in applied mathematics and mechanics from Moscow State University, Moscow, Russia, in 1991 and the Ph.D. degree in mechanical engineering from the University of Wisconsin, Madison, in 1997.

He has been on the Faculty of the University of California at Irvine (UCI), since 2000, where he is an Associate Professor with the Department of Mechanical and Aerospace Engineering, the Department of Electrical Engineering and Computer Sciences, and the Department of Biomedical Engineering. He is also Director of the UCI Micro-Systems Laboratory. After receiving the Ph.D. degree, he joined Berkeley Sensor and Actuator Center (BSAC) as a Postdoctoral Researcher. He then held research and consulting positions in several high-tech and venture companies. His professional interests, reflected in more than 100 publications, include solid-state sensors and actuators, MEMS-based neuroprosthetics, sensor-based intelligence, and control theory. He has received ten U.S. and international patents (14 are pending) on micromachined angle-measuring gyroscope, wide-bandwidth rate gyroscopes, design and fabrication of light manipulators and tunable optical filters, and hybrid surface micromachining processes.

Dr. Shkel has served on a number of editorial boards, including the IEEE/ ASME JouRnal of MicRoeleCtromeChaniCAL Systems and the International Journal on Smart Structures and Systems. He is a member of American Society of Mechanical Engineers (ASME), The International Society for Optical Engineers (SPIE), and the Institute of Navigation (ION). He received the 2006 Research Award of the UCI School of Engineering, the 2005 National Science Foundation (NSF) CAREER Award, the 2002 George E. Brown, Jr. Award, and a 2001 Fellowship from the Japanese Advanced Science Institute. He was Guest Editor for two special issues of the IEEE SENSORS JOURNAL, General Chair of the 2005 IEEE Sensors Conference, and is currently the Vice President of conferences of the IEEE Sensors Council. 